\title{
New Solutions of Peano's Differential Equation
}

\author{
Milan R. TASKović
}

\begin{abstract}
This paper gives sufficient conditions for new solutions of Peano's differential equation in the class of all lower continuous mappings. In this sense, this paper presents new fixed point theorems of Schauder type on lower transversal spaces. For the lower transversal space $(X, \rho)$ are essential the mappings $T: X \rightarrow X$ which are unbounded variation, i.e., if

$$
\sum_{n=0}^{\infty} \rho\left(T^{n} x, T^{n+1} x\right)=+\infty
$$

for arbitrary $x \in X$. On the other hand, for upper transversal spaces are essential the mappings $T: X \rightarrow X$ which are bounded variation!
\end{abstract}

\section{INTRODUCTION AND HISTORY}

Main fact of this paper is an investigation of solvability of the following initial value problem of the form

$$
x^{\prime}(t)=f(t, x(t)), \quad x\left(t_{0}\right)=y_{0},
$$

where $f$ is merely continuous or lower transversal continuous. In this sense I begin with the following essential facts on the transversal spaces and their further application for the solution of differential equation (De).

The possibility of defining such notions as limit and continuity in an arbitrary set is an idea which undoubtedly was first put forward by M. Fréchet in 1904, and developed by him in his famous thesis in 1906.

The simplest and most fruitful method which be proposed for such definitions was the introduction of the notion of distance.

But the greatest merit of Fréchet lies in the emphasis he put on three notions which were to play a fundamental part in all later developments of Functional Analysis: compactness, completeness, and separability.

In this sense, the notion of order, and the notion of new completeness, have each led to a fixed point statement. We now obtain geometric results of fixed points based on an interpley of these two notions as new notations in transversal upper and lower spaces.

2000 Mathematics Subject Classification. Primary: 47H10, Secondary: 54H25.

Key words and phrases. Fixed points, diametral $\varphi$-contractions, complete metric spaces, nonlinear conditions for fixed points, optimization. 
In connection with this, first, in Tasković [3] we introduced the concept of transversal (upper and lower) spaces as a natural extension of Fréchet's Kurepa's and Menger's spaces.

Let $X$ be a nonempty set. The function $\rho: X \times X \rightarrow \mathbb{R}_{+}^{0}:=[0,+\infty)$ is called an upper transverse on $X$ (or upper transversal) iff: $\rho[x, y]=$ $\rho[y, x], \rho[x, y]=0$ if and only if $x=y$, and if there is function $\psi:\left(\mathbb{R}_{+}^{0}\right)^{2} \rightarrow$ $\mathbb{R}_{+}^{0}$ such that

$$
\rho[x, y] \leq \max \{\rho[x, z], \rho[z, y], \psi(\rho[x, z], \rho[z, y])\}
$$

for all $x, y, z \in X$. An upper transversal space is a set $X$ together with a given upper transverse on $X$. The function $\psi$ in (As) is called upper bisection function.

On the other hand, the function $\rho: X \times X \rightarrow[0,+\infty]:=\mathbb{R}_{+}^{0} \cup\{+\infty\}$ is called a lower transverse on $X$ (or lower transversal) iff: $\rho[x, y]=\rho[y, x]$, $\rho[x, y]=+\infty$ if and only if $x=y$, and if there is a lower bisection function $d:[0,+\infty]^{2} \rightarrow[0,+\infty]$ such that

$$
\rho[x, y] \geq \min \{\rho[x, z], \rho[z, y], d(\rho[x, z], \rho[z, y])\}
$$

for all $x, y, z \in X$. A lower transversal space is a set $X$ together with a given lower transverse on $X$. The function $d$ in (Am) is called lower bisection function.

In recent years a great number of papers have presented results in the fixed point theory on miscellaneous spaces.

In this paper we begin with intensive development of the fixed point theory on transversal spaces, special, with development on lower transversal spaces.

Let $(X, \rho)$ be a lower transversal space and $T: X \rightarrow X$. We shall introduce the concept of DS-convergence in a space $X$; i.e., a lower transversal space $X$ satisfies the condition of DS-convergence (or $X$ is DS-complete) iff: $\left\{x_{n}\right\}_{n \in \mathbb{N}}$ is an arbitrary sequence in $X$ and $\sum_{i=1}^{\infty} \rho\left[x_{i}, x_{i+1}\right]=+\infty$ implies that $\left\{x_{n}\right\}_{n \in \mathbb{N}}$ has a convergent subsequence in $X$.

In connection with this, a lower transversal space $X$ satisfies the condition of orbitally DS-convergence (or $X$ is orbitally DS-complete) iff: $\left\{T^{n} x\right\}_{n \in \mathbb{N} \cup\{0\}}$ for $x \in X$ is an arbitrary iteration sequence in $X$ and

$$
\sum_{n=0}^{\infty} \rho\left[T^{n} x, T^{n+1} x\right]=+\infty \quad(\text { for } x \in X)
$$

implies that $\left\{T^{n} x\right\}_{n \in \mathbb{N} \cup\{0\}}$ has a convergent subsequence in $X$.

Annotation. We notice that in [9] Taskovic proved the following statement for a class of expansion mappings. Namely, if $(X, \rho)$ is an orbitally $D S$-complete lower transversal space, if $T: X \rightarrow X$, and if there exists a 
number $q>1$ such that

$$
\rho(T(x), T(y)) \geq q \rho(x, y)
$$

for each $x, y \in X$, then $T$ has a unique fixed point in the lower transversal space $X$.

Annotation. Let $\left(X, \rho_{X}\right)$ and $\left(Y, \rho_{Y}\right)$ be two lower transversal spaces and let $T: X \rightarrow Y$. We notice, from Taskovic [9], that $T$ be lower transversal continuous (or lower continuous) at $x_{0} \in X$ iff for every $\varepsilon>0$ there exists a $\delta>0$ such that the relation

$$
\rho_{X}\left[x, x_{0}\right]>\delta \quad \text { implies } \quad \rho_{Y}\left[T(x), T\left(x_{0}\right)\right]>\varepsilon .
$$

A typical first example of a lower transversal continuous mapping is the mapping $T: X \rightarrow X$ with property (1). Also, the lower transverse $\rho$ need not be lower transversal continuous; but, for an arbitrary metric function $r(x, y)$ the lower transverse of the form $\rho[x, y]:=1 / r(x, y)$ is a lower transversal continuous function. For further facts on the lower transversal continuous mappings see: Tasković [9].

In this sense, for any nonempty set $S$ in the lower transversal space $X$ the diameter of $S$ is defined by

$$
\operatorname{diam}(S):=\inf \{\rho[x, y]: x, y \in S\}
$$

it is a positive real number or $+\infty$, and $A \subset B \operatorname{implies} \operatorname{diam}(B) \leq \operatorname{diam}(A)$. The relation $\operatorname{diam}(S)=0$ holds if and only if $S$ is a one point set.

Elements of a lower transversal space will usually be called points. Given a lower transversal space $(X, \rho)$, with the bisection funkction $d$ and a point $z \in X$, the open ball of center $z$ and radius $r>0$ is the set

$$
d(B(z, r)):=\{x \in X: \rho[z, x]>r\}
$$

In this sense, we have the following form of convergence on the lower transversal spaces. The convergence $x_{n} \rightarrow x$ as $n \rightarrow \infty$ in the lower transversal space $(X, \rho)$ means that

$$
\rho\left[x_{n}, x\right] \rightarrow+\infty \quad \text { as } \quad n \rightarrow \infty,
$$

or equivalently, for every $\varepsilon>0$ there exists an integer $n_{0}$ such that the relation $n \geq n_{0}$ implies $\rho\left[x_{n}, x\right]>\varepsilon$.

The sequence $\left\{x_{n}\right\}_{n \in \mathbb{N}}$ in the lower transversal space $(X, \rho)$ is called transversal sequence (or lower Cauchy sequence) iff for every $\varepsilon>0$ there is an $n_{0}=n_{0}(\varepsilon)$ such that

$$
\rho\left[x_{n}, x_{m}\right]>\varepsilon \text { for all } n, m \geq n_{0} .
$$

Let $(X, \rho)$ be a lower transversal space and $T: X \rightarrow X$. We notice, from Tasković [9], that a sequence of iterates $\left\{T^{n}(x)\right\}_{n \in \mathbb{N}}$ in $X$ is said to be 
transversal sequence if and only if

$$
\lim _{n \rightarrow \infty}\left(\operatorname{diam}\left\{T^{k}(x): k \geq n\right\}\right)=+\infty .
$$

In this sense, a lower transversal space is called lower complete iff every transversal sequence converges.

Also, a space $(X, \rho)$ is said to be lower orbitally complete (or lower $T$-orbitally complete) iff every transversal sequence which is contained in the orbit $O(x):=\left\{x, T x, T^{2} x, \ldots\right\}$ for some $x \in X$ converges in $X$.

\section{Fixed POINTS ON LOWER TRANSVERSAL SPACES}

We are now in a position to formulate our main statements (Geometric statements of fixed point) in the following forms.

Theorem 1. Let $T$ be a self-map on an orbitally DS-complete lower transversal space $(X, \rho)$. Suppose that there exists a function $G: X \rightarrow \mathbb{R}_{+}^{0}$ such that

$$
\rho(x, T(x)) \geq G(T x)-G(x)
$$

for every $x \in X$. If $x \mapsto G(T x)$ is an upper semicontinuous function and if $G\left(T^{n} a\right) \rightarrow+\infty$ as $n \rightarrow \infty$ for some $a \in X$, then $T$ has a fixed point in $X$.

Proof. Let $x \in X$ be an arbitrary point in $X$. We can show then that the sequence of iterates $\left\{T^{n} x\right\}_{n \in \mathbb{N} \cup\{0\}}$ in $X$ satisfies the condition of $D S$ convergence. In this sense, from (A) we have

$$
\sum_{i=0}^{n} \rho\left[T^{i} x, T^{i+1} x\right] \geq G\left(T^{n+1} x\right)-G(x),
$$

and thus, from the conditions for functional $G$, as $n \rightarrow \infty$, we obtain the following fact:

$$
\sum_{i=0}^{\infty} \rho\left[T^{i} a, T^{i+1} a\right] \geq \lim _{n \rightarrow \infty}\left(G\left(T^{n+1} a\right)-G(a)\right)=+\infty .
$$

Hence, for the subsequence of iterates $\left\{T^{n(k)} a\right\}_{k \in \mathbb{N} \cup\{0\}}$ in $X$, by orbitally $D S$-completeness, there is $\xi \in X$ such that $T^{n(k)} a \rightarrow \xi(k \rightarrow \infty)$. Since $\xi \in X$, from (A), we obtain the following inequality in the form

$$
\rho(\xi, T(\xi)) \geq G(T \xi)-G(\xi) ;
$$

i.e., since $x \mapsto G(T x)$ is an upper semicontinuous function, we have

$$
\rho(\xi, T(\xi)) \geq \limsup _{k \rightarrow \infty} G\left(T^{n(k)+1} a\right)-G(\xi)=+\infty ;
$$

which means, by the properties of a lower transversal space, $T \xi=\xi$. The proof is complete.

In connection with the preceding statement, we now show that the following facts hold. 
Let $X$ be a topological space (or only a nonempty set), let $T: X \rightarrow X$ and let $A: X \times X \rightarrow \mathbb{R}_{+}^{0}$ be a given mapping. We shall introduce the concept of $D A$-variation in a space $X$, i.e., a topological space $X$ satisfies the condition of $D A$-variation iff: there exists a function $A: X \times X \rightarrow \mathbb{R}_{+}^{0}$ such that

$$
\sum_{n=0}^{\infty} A\left(T^{n} x, T^{n+1} x\right)=+\infty
$$

for arbitrary $x \in X$. In this case if (2) holds, we say and that $T: X \rightarrow X$ is unbounded variation or unbounded $A$-variation.

Lemma 1. Let $X$ be a nonempty set, $T: X \rightarrow X$, and let $A: X \times X \rightarrow \mathbb{R}_{+}^{0}$ be a given mapping. Then the following facts are mutually equivalent:

(a) $T$ is an unbounded variation mapping on a nonempty set $X$ in the sense of the mapping A.

(b) There is an unbounded function $G: X \rightarrow \mathbb{R}_{+}^{0}$ such that holds the following inequality in the form

$$
A\left(T^{n} x, T^{n+1} x\right) \geq G(T x)-G(x)
$$

for every $n \in \mathbb{N} \cup\{0\}$ and for every $x \in X$, where $G\left(T^{n} x\right) \rightarrow+\infty$ as $n \rightarrow \infty$.

(c) There is a nonnegative sequences of real functions in the form $x \mapsto$ $C_{n}(x, T x)$ such that the following inequality holds

$$
A\left(T^{n} x, T^{n+1} x\right) \geq C_{n}(x, T x)
$$

for every $n \in \mathbb{N} \cup\{0\}$ and for every $x \in X$, where $\sum_{n=0}^{\infty} C_{n}(x, T x)=+\infty$ for arbitrary $x \in X$.

Proof. For the proof of this facts, first, suppose that holds (a), then we define the function $G: X \rightarrow \mathbb{R}_{+}^{0}$ by,

$$
G(x)=\sum_{i=0}^{n} A\left(T^{i} x, T^{i+1} x\right) \quad \text { for } \quad x \in X ;
$$

and thus we have $G(T x)-G(x) \leq A\left(T^{n} x, T^{n+1} x\right)$, i.e., the condition (b) holds. If (b) holds, we obtain that holds and the following inequality

$$
A\left(T^{n} x, T^{n+1} x\right) \geq G\left(T^{n+1} x\right)-G\left(T^{n} x\right)
$$

for every $x \in X$ and for every $n \in \mathbb{N} \cup\{0\}$. We set $C_{n}(x, T x)=G\left(T^{n+1} x\right)-$ $G\left(T^{n} x\right)$ directly we obtain (c). Also, elementary, (a) is a consequence of (c). The proof is complete.

Annotation. Let $X$ be a topological space, let $T: X \rightarrow X$, and let $A: X \times X \rightarrow \mathbb{R}_{+}^{0}$ be a given mapping. We shall introduce the concept of $D S$-convergence in a space $X$, i.e., a topological space $X$ satisfies the condition of $D S$-convergence iff: $\left\{x_{n}\right\}_{n \in \mathbb{N}}$ is an arbitrary sequence in $X$ and 
$\sum_{i=1}^{\infty} A\left(x_{i}, x_{i+1}\right)=+\infty$ implies that $\left\{x_{n}\right\}_{n \in \mathbb{N}}$ has a convergent subsequence in $X$.

On the other hand, a topological space $X$ satisfies the following condition of orbitally $D S$-convergence iff: $\left\{T^{n} x\right\}_{n \in \mathbb{N} \cup\{0\}}$ for $x \in X$ is an arbitrary iteration sequence in $X$ and $\sum_{n=0}^{\infty} A\left(T^{n} x, T^{n+1} x\right)=+\infty$ (for $\left.x \in X\right)$ implies that $\left\{T^{n} x\right\}_{n \in \mathbb{N} \cup\{0\}}$ has a convergent subsequence in $X$.

We are now in a position to formulate our main geometric statements of fixed point on arbitrary topological spaces.

Theorem 1a. Let $T$ be a self-map on a topological space $X$ which is with the property of orbitally DS-convergence. Suppose that there exists a function $G: X \rightarrow \mathbb{R}$ such that

$$
A(x, T(x)) \geq G(T x)-G(x)
$$

for every $x \in X$. If $x \mapsto G(T x)$ is an upper semicontinuous function and if $A(a, b)=+\infty$ iff $a=b$ and if $G\left(T^{n} z\right) \rightarrow+\infty(n \rightarrow \infty)$ for some $z \in X$, then $T$ has a fixed point in $X$.

The proof of this statement is a totally analogous with the proof of the preceding Theorem 1 .

In connection with the preceding, we shall introduce the concept of lower topological space. In this sense, the function $A: X \times X \rightarrow[0,+\infty]$ is called a lower transverse on a nonempty set $X$ (or lower transversal) iff: $A(x, y)=+\infty$ if and only if $x=y$ for all $x, y \in X$.

A lower topological space $(X, A)$ is a topological space $X$ together with a given lower tranverse $A$ on $X$.

Otherwise, the function $A$ is called a semilower transverse on a nonempty set $X$ iff: $A(x, y)=+\infty$ implies $x=y$ for all $x, y \in X$. A semilower topological space $(X, A)$ is a topological space $X$ together with a given semilower transverse $A$ on $X$.

We notice, in connection with the preceding, that Theorem 1a de facto on lower topological spaces holds.

Theorem 1b. Let $T$ be a self-map on a semilower topological space $(X, A)$ which is with the property of DS-convergence. Suppose that there exists an unbounded upper semicontinuous function $G: X \rightarrow \mathbb{R}_{+}^{0}$ such that

$$
A(x, y) \geq G(y)-G(x)
$$

for all $x, y \in X$. Then $T$ has a fixed point in $X$.

Proof. From facts of this statement there exists a sequence $\left\{x_{n}\right\}_{n \in \mathbb{N}}$ in $X$ such that $G\left(x_{n}\right) \rightarrow+\infty$ as $n \rightarrow \infty$. On the other hand, from (N), we have

$$
\sum_{n=1}^{\infty} A\left(x_{n}, x_{n+1}\right) \geq \sum_{n=1}^{\infty}\left(G\left(x_{n+1}\right)-G\left(x_{n}\right)\right)=+\infty
$$


and thus, by $D S$-completeness, there is $\zeta \in X$ such that $x_{n(k)} \rightarrow \zeta(k \rightarrow \infty)$. Since $\zeta \in X$, from $(\mathrm{N})$, we obtain the following inequality in the form

$$
A(\zeta, T(\zeta)) \geq G(\zeta)-G(T \zeta)
$$

i.e., since $G$ is an upper semicontinuous function, we have the following inequality and equality of the form

$$
A(\zeta, T(\zeta)) \geq \limsup _{k \rightarrow \infty} G\left(x_{n(k)}\right)-G(T \zeta)=+\infty,
$$

which means, by properties of a semilower topological space, $T(\zeta)=\zeta$. The proof is complete.

For further results the following fact is essential. In this sense, we notice that the preceding statement we can modify in the following sense. Namely, the next statement follows from Theorem 1 as follows.

Theorem 1c Let $T$ be a self-map on a DS-complete lower transversal space $(X, \rho)$. Suppose that there exists an unbounded above function $G$ : $X \rightarrow \mathbb{R}_{+}^{0} \cup\{+\infty\}$ such that for any $x \in X$, with $x \neq T x$, there exists $y \in X \backslash\{x\}$ with property

$$
\rho(x, y) \geq G(y)-G(x),
$$

where $x \mapsto G(T x)$ is an upper semicontinuous function, then $T$ has a fixed point in $X$. (For further applications this statement is essential!)

A brief suitable proof of this statement based on Zorn's lemma may be found in Tasković [9].

General annotation. For further facts, in connection with the preceding problems of fixed point and transversal spaces, see: Tasković [2]-[9].

\section{Convexity on lower transversal spaces}

Let $X$ be a linear space over $\mathbb{K}(:=\mathbb{R}$ or $\mathbb{C})$. The mapping $x \mapsto\|x\|$ : $X \rightarrow \mathbb{R}$ is called an upper transversal seminorm (or upper seminorm) iff: $\|x\| \geq 0$ for every $x \in X,\|\lambda x\|=|\lambda|\|x\|$ for all $\lambda \in \mathbb{K}$ and $x \in X$, and if there is a function $\psi:(\mathbb{R} R)^{2} \rightarrow \mathbb{R} R:=[0,+\infty)$ such that

$$
\|x+y\| \leq \max \{\|x\|,\|y\|, \psi(\|x\|,\|y\|)\}
$$

for all $x, y \in X$.

Further, $x \mapsto\|x\|$ is called an upper transversal norm (or upper norm) iff in addition: $\|x\|=0$ if and only if $x=0$.

An upper transversal normed space $(X,\|\|$.$) over \mathbb{K}$ consists of a linear space $X$ over $\mathbb{K}$ together with an upper transversal norm $x \mapsto\|x\|$.

The function $\psi:(\mathbb{R} R)^{2} \rightarrow \mathbb{R} R$ in $(\mathrm{Nu})$ is called upper bisection function. From $(\mathrm{Nu})$ it follows, by induction, that there is a function 
$\mathcal{M}:(\mathbb{R} R)^{n} \rightarrow \mathbb{R} R$ such that

$$
\begin{gathered}
\left\|x_{0}-x_{n}\right\| \leq \\
\leq \max \left\{\left\|x_{0}-x_{1}\right\|, \ldots,\left\|x_{n-1}-x_{n}\right\|, \mathcal{M}\left(\left\|x_{0}-x_{1}\right\|, \ldots,\left\|x_{n-1}-x_{n}\right\|\right)\right\}
\end{gathered}
$$

for all $x_{0}, x_{1}, \ldots, x_{n} \in X$ and for any fixed integer $n \geq 2$.

It is easy to verify that the upper transversal normed linear space $X$ is a transversal upper space (see: Tasković [6]) with respect to the upper transverse $\rho: X \times X \rightarrow \mathbb{R} R$ defined by

$$
\rho[x, y]=\|x-y\| \quad \text { for all } \quad x, y \in X
$$

thus we obtain $\rho[x-z, y-z]=\rho[x, y]$ and $\rho[\lambda x, \lambda y]=|\lambda| \rho[x, y]$ for all $x, y, z \in X$ and for every scalar $\lambda \in \mathbb{K}$.

In this sense, an upper transversal normed space $X$ is said to be upper complete if it is upper complete as a transversal upper space. The upper convergence $x_{n} \rightarrow x(n \rightarrow \infty)$ means $\left\|x_{n}-x\right\| \rightarrow 0(n \rightarrow \infty)$.

We will in further denote by $\mathcal{B}\left(\mathbb{R}_{+}^{0}\right)$ the set of all upper bisection functions $\psi:(\mathbb{R})^{2} \rightarrow \mathbb{R}$ which are increasing satisfying $\psi(t, t) \leq t$ for every $t \in \mathbb{R}$.

In an former paper (Tasković: Math Japonica, 37 (1992), 367-372), have introduced the notion of general convex functions. A function $f: D \rightarrow \mathbb{R}$, where $\mathbb{R}$ denotes the real line and $D$ is a convex subset of $\mathbb{R}^{n}$, is said to be general convex iff there is a function $\psi:(f(D))^{2} \rightarrow \mathbb{R}$ such that

$(\operatorname{Max})$

$$
f(\lambda x+(1-\lambda) y) \leq \max \{f(x), f(x), \psi(f(x), f(y))\}
$$

for all $x, y \in D$ and for arbitrary $\lambda \in[0,1]$. We notice that the set of all convex and quasiconvex functions can be a proper subset of the set all general convex functions.

Let $X$ be a linear space over $\mathbb{K}$. The mapping $x \mapsto\|x\|: X \rightarrow[0,+\infty]$ is called a lower transversal seminorm (or lower seminorm) iff: $\|x\| \geq 0$ for every $x \in X,\|\lambda x\|=|f(\lambda)|\|x\|$ for all $\lambda \in \mathbb{K}$ and $x \in X$, where $f: \mathbb{K} \rightarrow \mathbb{K}$, and if there is a function $d:[0,+\infty]^{2} \rightarrow[0,+\infty]$ such that

$$
\|x+y\| \geq \min \{\|x\|,\|y\|, d(\|x\|,\|y\|)\}
$$

for all $x, y \in X$.

Further, $x \mapsto\|x\|$ is called a lower transversal norm (or lower norm) iff in addition: $\|x\|=+\infty$ if and only if $x=0$.

A lower transversal normed space $(X,\|\cdot\|)$ over $\mathbb{K}$ consists of a linear space $X$ over $\mathbb{K}$ together with a lower transversal norm $x \mapsto\|x\|$.

The function $d:[0,+\infty]^{2} \rightarrow[0,+\infty]$ in (Nl) is called lower bisection function. From $(\mathrm{Nl})$ it follows, by induction, that there is a function $\mathcal{D}$ : 
$[0,+\infty]^{n} \rightarrow[0,+\infty]$ such that

$$
\begin{gathered}
\left\|x_{0}-x_{n}\right\| \geq \\
\geq \min \left\{\left\|x_{0}-x_{1}\right\|, \ldots,\left\|x_{n-1}-x_{n}\right\|, \mathcal{D}\left(\left\|x_{0}-x_{1}\right\|, \ldots,\left\|x_{n-1}-x_{n}\right\|\right)\right\}
\end{gathered}
$$

for all $x_{0}, x_{1}, \ldots, x_{n} \in X$ and for any fixed integer $n \geq 2$.

It is easy to verify that the lower transversal normed linear space $X$ is a transversal lower space (see: Tasković [9]) with respect to the lower transverse $\rho: X \times X \rightarrow[0,+\infty]$ defined by

$$
\rho[x, y]=\|x-y\| \quad \text { for all } \quad x, y \in X ;
$$

thus we obtain $\rho[x-z, y-z]=\rho[x, y]$ and $\rho[\lambda x, \lambda y]=|f(\lambda)| \rho[x, y]$ for all $x, y, z \in X$ and for every scalar $\lambda \in \mathbb{K}$.

In this sense, the sequence $\left\{x_{n}\right\}_{n \in \mathbb{N}}$ in $(X,\|\|$.$) converges (or lower con-$ verges) to $x \in X$ if the sequence $\left\{x_{n}\right\}_{n \in \mathbb{N}}$ converges (or lower converges) in $(X, \rho)$, i.e., if

$$
\rho\left[x_{n}, x\right]=\left\|x_{n}-x\right\| \rightarrow+\infty \quad \text { as } \quad n \rightarrow \infty .
$$

In this sense, a lower transversal normed space $X$ is said to be lower complete (or complete) if it is lower complete as a transversal lower space.

We will, in further, denote by $\mathcal{D}([0,+\infty])$ the set of all lower bisection functions $d:[0,+\infty]^{2} \rightarrow[0,+\infty]$ which are increasing satisfying $d(t, t) \geq t$ for every $t \in[0,+\infty]$.

A function $f: D \rightarrow \mathbb{R}$, where $\mathbb{R}$ denotes the ral line in $D$ is a convex subset of $\mathbb{R}^{n}$, is said to be general concave iff there is a function $d:(f(D))^{2} \rightarrow \mathbb{R}$ such that

$$
f(\lambda x+(1-\lambda) y) \geq \min \{f(x), f(y), d(f(x), f(y))\}
$$

for all $x, y \in D$ and for arbitrary $\lambda \in[0,1]$. For this see: Tasković [4].

We notice that lower transversal norm $x \mapsto\|x\|$ is a general concave function. The proof is simple.

Lower bounded linear operators. Let $X$ and $Y$ be lower transversal normed spaces over $\mathbb{K}$. The linear operator $A: X \rightarrow Y$ is called lower bounded (or bounded) iff

$$
\inf _{x \in X \backslash\{0\}} \frac{\|A x\|}{\|x\|}>0 ;
$$

and, thus, the set $B(X, Y)$ of lower bounded linear operators from $X$ to $Y$ together with the operator lower norm of the form

$$
\|A\|:=\inf _{x \in X \backslash\{0\}} \frac{\|A x\|}{\|x\|}
$$

is a complete (lower complete) lower transversal normed space over $\mathbb{K}$.

Let $X$ and $Y$ be lower transversal normed spaces, then a map $f: M \subset$ $X \rightarrow Y$ is lower transversal continuous (or lower continuous) at $x \in M$ 
iff for every $\varepsilon>0$ there is a $\delta=\delta(\varepsilon)>0$ such that $\|f(x)-f(y)\|>\varepsilon$ whenever $y \in M$ and $\|x-y\|>\delta$.

Lower relatively compactness. Let $(X,\|\cdot\|)$ be a lower transversal normed space. A set $M$ in $X$ is lower bounded iff there is a number $r>0$ such that $\|x\| \geq r$ for all $x \in M$.

A set $M$ in $X$ is lower relatively compact (resp. lower compact) iff every sequence in $M$ contains a lower convergent subsequence (resp. the lower limit of which also belongs to $M$ ).

The set $M$ is lower dense in $X$ iff $\bar{M}=X$, i.e., for every $x \in X$ there exists a sequence $\left\{x_{n}\right\}_{n \in \mathbb{N}}$ in $M$ such that $x_{n} \rightarrow x$ as $n \rightarrow \infty$. This is equivalent to the condition that for every $x \in X$ and for every $\varepsilon>0$ there is a point $y \in M$ such that $\|x-y\|>\varepsilon$. The concept of denseness in lower transversal normed spaces covers many new approximation results.

Let $X$ and $Y$ are lower transversal normed spaces, then a map $f: M \subset$ $X \rightarrow Y$ is lower transversal continuous (or lower continuous) at $x \in M$ iff $x_{n} \rightarrow x$ as $n \rightarrow \infty$ implies $f\left(x_{n}\right) \rightarrow f(x)$ as $n \rightarrow \infty$.

Here it naturally is assumed that all of the $x_{n}$ belong to $M$. That $f$ is lower transversal continuous at $x$ then is equivalent to: for every $\varepsilon>0$ there is a $\delta(\varepsilon)>0$ such that

$$
\|f(x)-f(y)\|>\varepsilon \quad \text { whenever } \quad y \in M \quad \text { and } \quad\|x-y\|>\delta(\varepsilon) .
$$

Let $G$ be a nonempty bounded open set in $\mathbb{R}^{n}$. Then $L C(\bar{G})$ denotes the set of all real lower transversal continuous functions $f: \bar{G} \rightarrow \mathbb{R}$. For the special case where $G$ is a bounded open interval $(a, b)$ we write $L C[a, b]$ for $L C(\bar{G})$. In this sense, the set $M$ in $L C(\bar{G})$ is lower relatively compact iff:

(i) (lower uniformly boundedness)

$$
\inf _{f \in M}\left(\frac{1}{\sup _{x \in \bar{G}}|f(x)|}\right)>0
$$

(ii) (lower equicontinuity) for every $\varepsilon>0$ there is a $\delta(\varepsilon)>0$ such that the following fact holds that is

$$
\left(\sup _{f \in M} \mid f(x)-f(y)\right)^{-1}>\varepsilon \quad \text { whenever } \quad x, y \in \bar{G} \quad \text { and } \quad|x-y|^{-1}>\delta(\varepsilon) ;
$$

where here $\delta(\epsilon)$ is independent of $x, y$ and $f$.

The space $L C(\bar{G}, Y)$. Let $G$ be a nonempty bounded open set in $\mathbb{R}^{n}$ and let $\left(Y,\|\cdot\|_{Y}\right)$ be a lower transversal normed space over $\mathbb{K}$. We let $L C(\bar{G}, Y)$ denote the set of all lower transversal continuous functions $f: \bar{G} \rightarrow Y$. Then $L C(\bar{G}, Y)$ becomes a lower transversal normed space over $\mathbb{K}$ with the maximum norm of the form

$$
\|f\|=1 / \max _{x \in \bar{G}}\|f(x)\|_{Y} .
$$


In this sense we have the following statement of a Form of the ArzelàAscoli theorem for the lower transversal normed spaces: The set $M$ in $L C(\bar{G}, Y)$ is lower relatively compact iff: the set $\{f(x): f \in M\}$ is lower relatively compact in $Y$ for all $x \in \bar{G}$, and for every $x \in \bar{G}$ and every $\varepsilon>0$ there is a $\delta(\varepsilon, x)>0$ which is independent of the function $f$ such that

$\inf _{f \in M}\|f(x)-f(y)\|_{Y}>\varepsilon \quad$ whenever $\quad y \in \bar{G} \quad$ and $\quad\|x-y\|>\delta(\varepsilon, x)$.

A brief proof of this statement may be found in: Tasković [9]. This statement is essential for further facts on lower transversal normed spaces.

Lower compact operators. Let $X$ and $Y$ be lower transversal normed spaces and $T: D(T) \subset X \rightarrow Y$ an operator. The operator $T$ is called lower compact iff: $T$ is lower transversal continuous and $T$ maps lower bounded sets into lower relatively compact sets.

Typical example of lower compact operators on infinite dimensional lower transversal normed spaces is integral operator in the following form:

$$
(T x)(t)=\int_{a}^{t} K(t, s, x(s)) \mathrm{d} s \text { for all } t \in[a, b],
$$

where $K:[a, b] \times[a, b] \times[-r, r] \rightarrow \mathbb{K}(:=\mathbb{R}, \mathbb{C})$ is a continuous function. Set

$$
M:=\{x \in L C([a, b], \mathbb{K}):\|x\| \geq r\},
$$

where $\|x\|=1 / \max _{a \leq s \leq b}|x(s)|$ and $L C([a, b], \mathbb{K})$ is the space of all lower transversal continuous maps $x:[a, b] \rightarrow \mathbb{K}$.

In this sense, we will consider $T$ for $\mathbb{K}=\mathbb{R}$. The remaining case is treated similarly. The set $A=[a, b] \times[a, b] \times[-r, r]$ is compact, whence $K$ is bounded and uniformly continuous on $A$. Thus there is a number $\alpha$ such that $|K(t, s, x)| \leq \alpha$ for all $(t, s, x) \in A$, and for every $\varepsilon>0$ there is a $\delta(\varepsilon)>0$ such that $\left|K\left(t_{1}, s_{1}, x_{1}\right)-K\left(t_{2}, s_{2}, x_{2}\right)\right|<\varepsilon$ for all $\left(t_{i}, s_{i}, x_{i}\right) \in A$ and $i=1,2$ satisfying $\left|t_{1}-t_{2}\right|+\left|s_{1}-s_{2}\right|+\left|x_{1}-x_{2}\right|<\delta(\varepsilon)$. Let $z=T x$ and $x \in M$. Then

$$
\frac{1}{z(t)} \geq \frac{1}{\left|\int_{a}^{t} K(t, s, x(s)) \mathrm{d} s\right|} \geq \frac{1}{(b-a) \alpha}
$$

for all $t \in[a, b]$. Furthermore, for $\left|t_{1}-t_{2}\right| \leq \min \{\delta(\varepsilon), \varepsilon\}$, we obtain the following inequalities of the form

$$
\begin{gathered}
\frac{1}{\left|z\left(t_{1}\right)-z\left(t_{2}\right)\right|}=\left|\int_{a}^{t_{1}} K\left(t_{1}, s, x(s)\right) \mathrm{d} s-\int_{a}^{t_{2}} K\left(t_{2}, s, x(s)\right) \mathrm{d} s\right|^{-1}= \\
=\left|\int_{a}^{t_{1}}\left(K\left(t_{1}, s, x(s)\right)-K\left(t_{2}, s, x(s)\right)\right) \mathrm{d} s-\int_{t_{1}}^{t_{2}} K\left(t_{2}, s, x(s)\right) \mathrm{d} s\right|^{-1} \geq \\
\geq \frac{1}{(b-a) \varepsilon+\left|t_{1}-t_{2}\right| \alpha} \geq \frac{1}{((b-a)+\alpha) \varepsilon} ;
\end{gathered}
$$


and thus, the preceding two inequalities are uniformly true for $z=T x$ with arbitrary $x \in M$. By the Form of the Arzelà-Ascoli theorem (for lower transversal spaces), the set $T(M)$ is lower relatively compact.

On the other hand, the operator $T$ is lower transversal continuous on $M$. To see this, let $\left\{x_{n}\right\}_{n \in \mathbb{N}}$ be a sequence in $M$ with $\left\|x_{n}-x\right\| \rightarrow+\infty$ as $n \rightarrow \infty$, i.e., the functions $x_{n}(t)$ lower converge uniformly on $[a, b]$ to $x(t)$. Set $z_{n}=T\left(x_{n}\right)$ and $z=T(x)$. Then

$$
\begin{gathered}
\left\|z-z_{n}\right\|=\frac{1}{\max _{a \leq t \leq b}\left|z(t)-z_{n}(t)\right|}= \\
=\left(\max _{a \leq t \leq b}\left|\int_{a}^{t}\left(K(t, s, x(s))-K\left(t, s, x_{n}(s)\right)\right) \mathrm{d} s\right|\right)^{-1} \rightarrow+\infty \text { as } n \rightarrow \infty ;
\end{gathered}
$$

and thus, all the preceding facts together imply the lower compactness of $T$. The prove this fact is complete.

The set $C$ in linear space is lower convex if for $x, y \in C$ and $\lambda \in[1,2]$ implies that $\lambda x+(1-\lambda) y \in C$. The lower transversal space $(X, \rho)$ is called lower convex (or transversal lower convex) if for any two different points $x, y \in X$ there is a point $z \in X(z \neq x, y)$ such that

$$
\rho[x, y]+\rho[y, z]=\frac{9}{2} \rho[x, z] .
$$

In connection with this, if $C \subset X$ is a lower convex set of transversal lower normed space $X$, then $C$ also and transversal lower convex space with $\rho[x, y]=1 /\|x-y\|$, for the classical norm $\|\cdot\|$, because for any two different points $x, y \in C$ there is a point $z:=(3 y-x) / 2 \in C(z \neq x, y)$ such that $(\mathrm{Cd})$ holds. For further facts on lower transversal normed spaces see: Tasković [9].

We are now in a position to formulate the following general statements which are a based for geometry (lower convexity) of lower transversal spaces. The following statements are very connection with the famous Schauder's problem (Scottish book, problem 54), from: Tasković [8].

Proposition 1. Let $C$ be a nonempty lower convex compact subset of a linear topological space $X$ and suppose $T: C \rightarrow C$ is a lower transversal continuous mapping. Then $T$ has a fixed point in $C$.

A suitable proof of this statement may be found in Tasković [9]. Also, a brief proof of this statement we can give and from the preceding facts of this paper.

Proposition 2. Suppose that $C$ is a nonempty lower convex lower compact subset of $\mathbb{R}^{n}$, and that $T: C \rightarrow C$ is a lower transversal continuous mapping. Then $T$ has a fixed point in $C$.

We can now formulate Proposition 2 in a manner valid for all transversal lower normed linear space. 
Proposition 3. Let $C$ be a nonempty, lower compact, lower convex subset of a transversal lower normed space $X$, and suppose $T: C \rightarrow C$ is a lower transversal continuous operator. Then $T$ has a fixed point in $C$.

We notice that Propositions 2 and 3 are directly consequences of Proposition 1. A brief suitable proof of Proposition 3 may be found in Tasković [9].

On the other hand, in connection with the preceding facts, the set $C$ in linear space is convex if for $x, y \in C$ and $\lambda \in[0,1]$ implies $\lambda x+(1-\lambda) y \in C$.

The lower transversal space $(X, \rho)$ is called $D$-convex (or transversal $D$ convex $)$ if for two different point, $x, y \in X$ there is a point $z \in X(z \neq x, y)$ such that

$$
\rho[x, y]+\rho[y, z]=\rho[x, z] .
$$

In connection with this, if $C \subset X$ is a convex set of a transversal lower normed space $X$, then $C$ also and transversal $D$-convex space with $\rho[x, y]=$ $1 /\|x-y\|$, for the classical norm $\|\cdot\|$, because for any two different points $x, y \in C$ there is a point

$$
z:=\frac{3-\sqrt{5}}{2} y+\frac{\sqrt{5}-1}{2} x \in C \quad(z \neq x, y)
$$

such that (Dc) holds. For futher facts see: Tasković [9].

Proposition 4. Let $C$ be a nonempty convex compact subset of a linear topological space $X$ and suppose $T: C \rightarrow C$ is a lower transversal continuous mapping. Then $T$ has a fixed point in $C$.

A proof of this statement we can give from the preceding facts of this paper. The proof of this statement is similary with the proof of the former Proposition 1.

Proposition 5. Suppose that $C$ is a nonempty $D$-convex lower compact subset of $\mathbb{R}^{n}$, and that $T: C \rightarrow C$ is a lower transversal continuous mapping. Then $T$ has a fixed point in $C$.

¿From Proposition 4 we can now formulate Proposition 5 in a manner valid for all transversal lower normed spaces.

Proposition 6. Let $C$ be a nonempty, lower compact, D-convex subset of a transversal lower normed space $X$, and suppose $T: C \rightarrow C$ is a lower transversal continuous operator. Then $T$ has a fixed point in $C$.

We notice that Propositions 5 and 6 are directly consequences of Proposition 4. A brief suitable proof of Proposition 6 may be found in Tasković [9].

A direct equivalent translation of Propositions 3 and 6 to lower compact operators is the following result. 
Proposition 7. Let $C$ be a nonempty, closed, lower bounded, D-convex (or lower convex) subset of a lower complete transversal lower normed space $X$, and suppose $T: C \rightarrow C$ is a lower compact operator. Then $T$ has a fixed point in $C$.

A brief proof of this statement may be found in Tasković [9]. This form of this statement is useful for applications.

Open problem. Does every lower continuous mapping of compact set $C \subset X$ into itself in linear topological space $X$ has a fixed point in $C$, where $C$ with the property that: $\lambda x+(1-\lambda) y \in C$ for all $x, y \in C$ and $\lambda \in[n, n+1]$ for an arbitrary fixed number $n \in \mathbb{N}$ !?

We notice that the cases $n=0$ and $n=1$, of this problem, are solve via Propositions 1 and 4 of this paper.

Lemma 2. Let $(X, \rho)$ be a lower transversal space. If $C$ is a transversal $D$-convex or lower transversal convex set and if $T: C \rightarrow C$, then there exists a function $G: C \rightarrow \mathbb{R}_{+}^{0} \cup\{+\infty\}$ such that $T$ with the property (Lc).

Proof. Let $a \in C$ be a fixed element and let $x \in C$ be an arbitrary point with $x \neq a$. First, since $C$ is a $D$-convex set in $X$, it follows from definition that for $a \in C$ and for all $x \in C \backslash\{a\}$ there exists a point $y \neq a, x$ in $C$ such that $\rho[a, x]+\rho[x, y]=\rho[a, y]$. Hence, we have, also and the following inequality of the form

$$
3 \rho[x, y] \geq \rho[x, y]=\rho[a, y]-\rho[a, x],
$$

for every $x \in C \backslash\{a\}$. On the other hand, if $C$ is a transversal lower convex set, then again holds (3) for every $x \in C \backslash\{a\}$. We notice that (3) simply holds and for $x=a$. Hence, from inequality (3) define function $G: C \rightarrow$ $\mathbb{R} R \cup\{+\infty\}$ such that

$$
G(x)=3^{-1} \rho[a, x] \text { for } \quad x \in C .
$$

Then, clearly, from (3) and (4) we have for any $x \in C$ that there exists $y \neq x$ in $C$ such that $\rho[x, y] \geq G(y)-G(x)$. Thus for any $x \in C$ with $x \neq T x$ there exists $y \in C \backslash\{x\}$ such that (Lc). Hence, it follows that $T$ is with the property (Lc) and the proof is complete.

Lemma 3. Let $X$ be a linear space. If $C$ is a D-convex on lower convex set in $X$ and if $T$ is a map of $C$ into itself, then there exists a continuous function $G: C \rightarrow \mathbb{R} R \cup\{+\infty\}$ such that $T$ is a with the property $(\mathrm{Lc})$.

Proof. Consider the $D$-convex (or lower convex) set $C$ of the linear space $X$ as a lower quasi-transversal space with the quasi lower transverse $q$, where $q: C \times C \rightarrow \mathbb{R} R \cup\{+\infty\}$ defined by

$$
q(x, y)=\left\{\begin{aligned}
+\infty, & \text { for } \quad x=y \\
\min \{K(x), K(y)\}, & \text { for } \quad x \neq y
\end{aligned}\right.
$$


for a strictly convex continuous function $K: C \rightarrow \mathbb{R} R \cup\{+\infty\}$. Then it is easy to see that $q$ is a lower quasi-transverse, i.e., that for all $x, y, z \in C$ we have: $q(x, y)=q(y, x)$, and

$$
q(x, y) \geq \min \{q(x, z), q(z, y), d(q(x, z), q(z, y))\}
$$

for some $d:(\mathbb{R} R \cup\{+\infty\})^{2} \rightarrow \mathbb{R} R \cup\{+\infty\}$, and that $x=y$ implies $q(x, y)=+\infty$.

On the other hand, if $q(x, y)=+\infty$ and $x \neq y$, i.e., if $K(x)=K(y)=$ $+\infty$, then since $K$ is a strictly convex function, we obtain the following fact

$$
+\infty=\frac{K(x)+K(y)}{2}>K\left(\frac{x+y}{2}\right)=+\infty,
$$

which is a contradiction. Consequently $x=y=(x+y) / 2$, i.e., $x=y$. Thus $q(x, y)=+\infty$ implies $x=y$, i.e., $q$ is a continuous lower transverse on $C$.

Applying Lemma 2 to this case, we obtain then that there exists a continuous function $G: C \rightarrow \mathbb{R} R \cup\{+\infty\}$ defined by $G(x)=3^{-1} q(x, y)$ such that $T$ is with the property $(\mathrm{Lc})$. The proof is complete.

Proof of Proposition 1. From Lemma 3 and the preceding facts there exists a continuous function $G: C \rightarrow \mathbb{R} R \cup\{+\infty\}$ such that $T$ with the property (Lc). Since $T$ is a lower continuous mapping, the function $x \mapsto$ $G(T x)$ is an upper semicontinuous function. The set $C$ is a compact in the space $X$ and thus $C$ satisfies the condition of $D S$-completeness. It is easy to see that $T$ satisfies all the required hypotheses in Theorem 1c. Hence, it follows from Theorem 1c, that $T$ has a fixed point in $C$. The proof is complete.

\section{Application for Peano's differential equation}

Now we shall show how Proposition 3 and 6, i.e., Proposition 7, can be applied for investigation of solvability of the initial value problem of the form

$$
x^{\prime}(t)=f(t, x(t)), \quad x\left(t_{0}\right)=y_{0},
$$

where $f$ is merely continuous or lower transversal continuous. In this sense the following result holds.

Theorem 2. Let there be given real numbers $t_{0}$ and $y_{0}$ and the following rectangle of the form

$$
Q_{b}:=\left\{(t, x) \in \mathbb{R}^{2}:\left|t-t_{0}\right| \leq 1 / a,\left|x-y_{0}\right| \leq 1 / b\right\},
$$

where $a$ and $b$ are fixed positive numbers. Suppose that $f: Q_{b} \rightarrow \mathbb{R}$ is continuous or lower transversal continuous and bounded with

$$
|f(t, x)| \leq K \quad \text { for all } \quad(t, x) \in Q_{b},
$$


and fixed $K>0$. For $m=\min \{1 / a, 1 / b K\}$ then the initial value problem (De) has a lower transversal continuously differentiable solution on the closed interval of the form $\left[t_{0}-m, t_{0}+m\right]:=I$.

In further let $L C\left[t_{0}-m, t_{0}+m\right]$ denote the space of all lower transversal continuous functions $x(t)$ where the lower norm $\|\cdot\|$ in the form

$$
\|x\|=\frac{1}{\max _{t \in I}|x(t)|} .
$$

Proof of Theorem 2. For the proof of this statement we first replace (De) by the integral equation in the form

$$
x(t)=y_{0}+\int_{t_{0}}^{t} f(s, x(s)) \mathrm{d} s,
$$

and next write this as the operator equation $x=T x$ for $x \in M \subset X$, where $M=\left\{x \in X:\left\|x-y_{0}\right\| \geq b\right\}$.

The set $M$ is closed, lower bounded, and $D$-convex of transversal lower normed space $X:=L C\left[t_{0}-m, t_{0}+m\right]$. We notice that $T(M) \subset M$, i.e., $T$ maps $M$ into $M$. For if $x \in M$, then $\left\|x-y_{0}\right\| \geq b$, and hence $\left|x(t)-y_{0}\right| \leq 1 / b$ for all $t \in I$. Thus,

$$
\left\|T x-y_{0}\right\|=\frac{1}{\max _{t \in I}\left|\int_{t_{0}}^{t} f(s, x(s)) \mathrm{d} s\right|} \geq \frac{1}{m K} \geq b ;
$$

therefore, $T x \in M$. The operator $T: M \rightarrow M$ is lower compact by the preceding typical example. Now, Proposition 7 implies the existence of a solution $x=T x, x \in M$. The proof is complete.

In this part, also, our goal is to generalize the preceding Form of Peano's theorem to equations of the form

$$
x^{\prime}(t)=f(t, x(t)), \quad x\left(t_{0}\right)=y_{0},
$$

where $x(t)$ lies in a lower transversal normed space $Y$. In the special case where $Y=\mathbb{R}^{p},(5)$ is the system

$$
\xi_{i}^{\prime}(t)=f_{i}(t, x(t)), \quad \xi_{i}\left(t_{0}\right)=\eta_{i 0}, \quad i=1, \ldots, n ;
$$

where $x(t)=\left(\xi_{1}(t), \ldots, \xi_{n}(t)\right)$, of $n$ ordinary differential equations. For continuous $f_{i}$ we obtain the equivalent system of integral equations

$$
\xi_{i}(t)=\eta_{i 0}+\int_{t_{0}}^{t} f_{i}(s, x(s)) \mathrm{d} s, \quad i=1, \ldots, n .
$$

As our lower transversal normed space we choose the set of all $x(t)$ for which the components $\xi_{i}(t)$ are lower continuous on $\left[t_{0}-m, t_{0}+m\right]$. For the lower norm on $Y$ we use

$$
\|x\|_{Y}:=\frac{1}{\max _{1 \leq i \leq n}\left(\max _{a \leq t \leq b}\left|\xi_{i}(t)\right|\right)}
$$


then, in complete analogy with Theorem 2 one shows the existence of solutions for some $m$ and for continuous $f_{i}$ in the class of all lower continuous functions.

In contrast to former fact that $f$ is continuous, or lower transversal continuous, we now require $f$ to be a lower compact mapping.

Theorem 3 (Generalized Theorem 2). Let $Y$ be a lower complete lower transversal normed space with a bisection function $d \in \mathcal{D}([0,+\infty])$, let $t_{0} \in$ $\mathbb{R}$ and $y_{0} \in Y$, and

$$
Q_{b}:=\left\{(t, y) \in \mathbb{R} \times Y:\left|t-t_{0}\right| \leq 1 / a,\left\|y-y_{0}\right\|_{Y} \leq 1 / b\right\},
$$

for fixed numbers $0<a, b<\infty$. Suppose that $f: Q_{b} \rightarrow Y$ is a lower compact map and that $\|f(t, y)\|_{Y} \leq K$ for all $(t, y) \in Q_{b}$ with fixed $K>0$. If we set $m=\min \{1 / a, 1 / b K\}$, then (5) has a lower continuously differentiable solution on $\left[t_{0}-m, t_{0}+m\right]:=I$.

Proof. We set $X:=L C\left(\left[t_{0}-m, t_{0}+m\right], Y\right)$ for $0<m<+\infty$, i.e., $X$ is the space of all lower continuous functions $x:\left[t_{0}-m, t_{0}+m\right] \rightarrow Y$, where as a lower norm, we choose

$$
\|x\|_{X}:=\max _{t \in\left[t_{0}-m, t_{0}+m\right]}\|x(t)\|_{Y}
$$

where $\|x\|_{Y}:=\left(\max _{t \in I}|x(t)|\right)^{-1}$ and, where $M:=\left\{x \in X:\left\|x-y_{0}\right\|_{X} \geq b\right\}$ is a ball (lower) in $X$. Define $z(t)$ to be the right side of the integral equation

$$
x(t)=y_{0}+\int_{t_{0}}^{t} f(s, x(s)) \mathrm{d} s:=z(t),
$$

and set $T(x)=z$. Then, this equation corresponds to the fixed point problem $x=T(x)$ for $x \in M \subset X$, and is also equivalent to (5). Since $\left\|x-y_{0}\right\|_{X} \geq b$ and $\left\|z-y_{0}\right\|_{X} \geq 1 / m K \geq b$ and

$$
\left\|z\left(t_{1}\right)-z\left(t_{2}\right)\right\|_{Y} \geq \frac{1}{K\left|t_{1}-t_{2}\right|}
$$

for all $t_{1}, t_{2} \in\left[t_{0}-m, t_{0}+m\right]$; if we now set

$$
A(t):=y_{0}+\left(t-t_{0}\right) \overline{\operatorname{conv}}\left\{f(s, x(s)): s \in\left[t_{0}-m, t_{0}+m\right]\right\},
$$

where $\overline{\mathrm{conv}}$ denote closed convex hull, then $z(t) \in A(t)$ and $T(M) \subset M$.

The set $T(M)$ is lower relatively compact in $X$, because for $(7)$ shows that the functions $z \in T(M)$ are lower uniformly continuous functions, and for all $t \in\left[t_{0}-m, t_{0}+m\right]$, their values lie in the lower compact set $A(t)$. Then, the Form of Arzelà-Ascoli theorem implies that $T(M)$ is lower relatively compact. 
On the other hand, the operator $T$ is lower continuous on $M$. To see this, let $x_{n} \rightarrow x$ in $X$ as $n \rightarrow \infty$. By (6), we have

$$
\left\|T x_{n}-T x\right\|_{X} \geq m \inf _{s \in\left[t_{0}-m, t_{0}+m\right]}\left\|f\left(s, x_{n}(s)\right)-f(s, x(s))\right\|_{Y} \rightarrow+\infty
$$

as $n \rightarrow \infty$. For if it did not, there would be an $\varepsilon_{0}>0$ and a sequence, denoted for brevity by $\left(s_{n}\right)$ in $\left[t_{0}-m, t_{0}+m\right]$ for which

$$
\left\|f\left(s_{n}, x_{n}\left(s_{n}\right)\right)-f\left(s_{n}, x\left(s_{n}\right)\right)\right\|_{Y} \leq \varepsilon_{0} ;
$$

then there is a subsequence, again denoted by $\left(s_{n}\right)$ and an $s_{0}$ such that $s_{n} \rightarrow s_{0}$ as $n \rightarrow \infty$, and

$$
\begin{aligned}
& \left\|x_{n}\left(s_{n}\right)-x\left(s_{0}\right)\right\|_{Y} \geq \min \left\{\left\|x_{n}\left(s_{n}\right)-x\left(s_{n}\right)\right\|_{Y},\left\|x\left(s_{n}\right)-x\left(s_{0}\right)\right\|_{Y},\right. \\
& \left.d\left(\left\|x_{n}\left(s_{n}\right)-x\left(s_{n}\right)\right\|_{Y},\left\|x\left(s_{n}\right)-x\left(s_{0}\right)\right\|_{Y}\right)\right\} \geq \\
& \geq \min \left\{\left\|x_{n}\left(s_{n}\right)-x\left(s_{n}\right)\right\|_{Y},\left\|x\left(s_{n}\right)-x\left(s_{0}\right)\right\|_{Y},\right. \\
& \left.\min \left\{\left\|x_{n}\left(s_{n}\right)-x\left(s_{n}\right)\right\|_{Y},\left\|x\left(s_{n}\right)-x\left(s_{0}\right)\right\|_{Y}\right\}\right\}= \\
& =\min \left\{\left\|x_{n}\left(s_{n}\right)-x\left(s_{n}\right)\right\|_{Y},\left\|x\left(s_{n}\right)-x\left(s_{0}\right)\right\|_{Y}\right\} \rightarrow+\infty \quad \text { as } n \rightarrow \infty \text {. }
\end{aligned}
$$

In this sense, we note that $\left\{x_{n}(s)\right\}_{n \in \mathbb{N}}$ is lower uniformly convergent, $x(t)$ is lower continuous, and $f$ is lower continuous, therefore both $f\left(s_{n}, x_{n}\left(s_{n}\right)\right)$ and $f\left(s_{n}, x\left(s_{n}\right)\right)$ lower converge to $f\left(s_{0}, x\left(s_{0}\right)\right)$ as $n \rightarrow \infty$, contradicting (8).

Apply the Proposition 7 to obtain the existence of a fixed point of $T$ on $M$. The proof is complete.

Aannotation. The preceding facts on lower compact operators can play a central role in nonlinear functional analysis. Their importance stems from the fact that many results on lower continuous operators on $\mathbb{R}^{n}$ carry over to transversal lower normed spaces when "lower continuous" is replaced by "lower compact".

In connection with this, as well-known, compact and completely continuous operators occur is many problems of classical analysis. In the nonlinear case, the first comprehensive research on compact operators with numerous applications to partial differential equations (both linear and nonlinear) was due to J. Schauder: Zur Theorie stetiger Abbildungen in Funktionalräumen, Math. Zeit., 26 (1927), 47-65.

Literature on applications of the Schauder theorem to nonlinear problems is extensive. The first topological proof of the Peano theorem is due to G. D. Birkhoff and O. D. Kellogg: Invariant points in function space, Trans. AMS, 23 (1922), 95-115.

Juliusz Schauder in 1930 brief: "The problem which this work brings to a certain conclusion was first investigated by Messrs. Birkhoff and Kellogg 
in 1922. These authors already recognized the correctness of the fixed point theorem in simplest function spaces, but for each individual function spaces, the proof had to be carried anew from the beginning".

We notice that the fundamental idea of applying fixed point results to produce theorems in analysis is due to H. Poincaré: Sur certaines solutions particulières du problème des trois corps, Bull. Astronom., 1 (1884), 65-74.

\section{REFERENCES}

[1] G. Peano: Démonstration de l'intégrabilité des équations différentielles ordinaires, Math. Ann., 37 (1890), 182-228.

[2] M. R. Tasković: A characterization of the class of contraction type mappings, Kobe J. Math., 2 (1985), 45-55.

[3] M. R. Tasković: Transversal spaces, Math. Moravica, 2 (1998), 133-142.

[4] M. R. Tasković: Nonlinear Functional Analysis, Second Book: Monographs - Global Convex Analysis - General convexity, Variational methods and Optimization, Zavod za udžbenike i nastavna sredstva i Vojnoizdavački zavod, Beograd 2001, (in Serbian), 1223 pages.

[5] M. R. Tasković: Transversal intervally spaces, Math. Moravica, 7 (2003), 87-102.

[6] M. R. Tasković: Survey on transversal normed spaces, Math. Moravica, 7 (2003), 149-170.

[7] M. R. Tasković: Fixed points on transversal edges spaces, Math. Moravica, 7 (2003), $171-182$.

[8] M. R. Tasković: On Schauder's 54th problem in Scottish book revisited, Math. Moravica, 6 (2002), 119-126.

[9] M. R. Tasković: Theory of transversal point, spaces, and forks; Monographs of a new mathematical theory, Beograd 2004, 1000 pages, (In Serbian), to appear.

Milan R. Tasković

FACUlty of Mathematics

11000 Belgrade, P.O. Box 550

SERBIA

Home Address:

Milan R. Tasković

Nehruova 236

11070 BELGRADE

SERBIA

E-mail address: andreja@predrag.us 